\title{
Estudio de susceptibilidad in vitro de Enterococcus spp
}

\author{
CHRYSTAL JULIET L. ${ }^{1}$
}

In vitro SUSCEPTIBILITY TESTING OF Enterococcus spp

Key words: Enterococcus, In vitro susceptibility, Vancomycin resistance.

El género bacteriano Enterococcus fue creado en 1980, retirándolo del género Streptococcus al cual pertenecía como parte del grupo $\mathrm{D}$, por diferencias genéticas. Se describen 16 especies (Tabla 1), siendo E. faecalis el más frecuentemente aislado de infecciones humanas $(95 \%$ en nuestro medio) y E. faecium en menor proporción $(5 \%)$.

Forman parte de la flora comensal intestinal humana y también de otros mamíferos, aves e insectos. Son capaces de sobrevivir en medios poco enriquecidos como agua y suelo, alimentos; en hospitales sobreviven en las manos de los portadores, fuentes y superficies inanimadas durante 24 horas. Son causantes en la mayoría de los casos, de infecciones endógenas, entre ellas, infección del tracto urinario (la más frecuente), endocarditis infecciosa, bacteremias e infecciones intrahospitalarias (IIH) asociadas

Tabla 1. Especies del género Enterococcus

$$
\mathrm{N}=\mathbf{1 6}
$$

\begin{tabular}{ll}
\hline S. avium & S. gallinarum \\
S. calcorum & S. hirae \\
S. casseliflavus & S. malodoratus \\
S. columbae & S. mundtii \\
S. dispar & S. pseudoavium \\
S. durans & S. raffinosus \\
S. faecalis & S. saccharolyticus \\
S. faecium & S. sulfureus \\
\hline
\end{tabular}

${ }^{1}$ Laboratorio de Microbiología. Hospital Del Salvador. a procedimientos como instalación de catéteres vasculares, urinarios y neuroquirúrgicos entre otros.

En Chile, durante 1999, Enterococcus fue responsable de $2,8 \%$ del total de las IIH notificadas con agente etiológico aislado; $2,7 \%$ de las ITU, 3,8\% de las bacteremias en general, $5,3 \%$ de las bacteremias asociadas a catéter vascular y menos del $1 \%$ de las infecciones de herida operatoria.

La importancia de este género radica en su alta resistencia natural a múltiples antimicrobianos y a su capacidad de adquirir resistencia a otros (Tabla 2), incluida vancomicina, en ocasiones única alternativa de tratamiento en infecciones severas por Enterococcus resistentes a ampicilina, y ampliamente utilizada en las últimas dos décadas en la terapia de infeccio-

Tabla 2. Tipos de resistencia en Enterococcus spp

\begin{tabular}{|c|c|}
\hline Natural a: & Adquirida a: \\
\hline $\begin{array}{l}\text { - } \text { Cefalosporinas } \\
\text { - Oxacilinas } \\
\text { - } \text { Clindamicina } \\
\text { - } \text { Lincomicina } \\
\text { - } \text { Aminoglucósidos } \\
\text { (bajo nivel) }\end{array}$ & $\begin{array}{l}\text { - Ampicilina-penicilina } \\
\text { - Cloranfenicol } \\
\text { - Eritromicina } \\
\text { - Tetraciclinas } \\
\text { - Quinolonas } \\
\text { - Vancomicina-teicoplanina } \\
\text { - Nitrofurantoína } \\
\text { - Aminoglucósidos (alto nivel) }\end{array}$ \\
\hline
\end{tabular}


nes por Staphylococcus aureus resistentes a cloxacilina.

El antimicrobiano de elección en las infecciones por Enterococcus es ampicilina, la que tiene un efecto inhibidor y no lítico. Para lograr un efecto bactericida en infecciones graves como endocarditis, bacteremias o meningitis, se recomienda asociar un aminoglucósido, siempre que la resistencia a este último sea de bajo nivel: $<500 \mu \mathrm{g} / \mathrm{ml}$ para gentamicina y $<2.000$ $\mu \mathrm{g} / \mathrm{ml}$ para estreptomicina.

La susceptibilidad natural de E. faecalis a ampicilina es mayor (CIM entre 0,5 y $4 \mu \mathrm{g} / \mathrm{ml}$ ) que $E$. faecium (4 a $8 \mu \mathrm{g} / \mathrm{ml})$. Ambas especies pueden adquirir resistencia de dos tipos a este antibacteriano:

Bajo nivel: CIM de 8 a $32 \mu \mathrm{g} / \mathrm{ml}$, determinada por una mayor producción de PBP 5 con menor afinidad a ampicilina y Alto nivel: CIM > $64 \mu \mathrm{g} / \mathrm{ml}$, que se debe a la producción de $B$ lactamasas, lo cual es inhabitual y se ha descrito sólo en E. faecalis. La producción de PBP 5, mecanismo más frecuente de resistencia, presente en E. faecium, se asocia en esta especie a resistencia a vancomicina tipo Van B, transferible(trasposon 5382, muy expandido enE.U.A.).

La resistencia adquirida a vancomicina presenta diferentes genotipos y sus características se expresan en la Tabla 3.

\section{Epidemiología de Enterococcus resistente a vancomicina (ERV)}

En 1986 se describieron las primeras cepas de Enterococcus resistentes a vancomicina (ERV) en Inglaterra; posteriormente, en este mismo país, se describió un incremento de $50 \%$ por año de las infecciones por este agente en algunos hospitales. En E.U.A. se comunicó un aumento de las IIH causadas por este agente, desde 0,4 a $23 \%$ en unidades de cuidados intensivos (UCIs) y desde 0,3 a $15 \%$ en otros servicios clínicos, entre 1989 y 1997.

Estos antecedentes motivaron a la Comisión Nacional de Infecciones Intrahospitalarias del MINSAL de nuestro país, en mayo del 2000, a iniciar mediante una Norma (Circular 4/C28 del 09/05/2000, Figura 1), un sistema de vigilancia de colonización intestinal por ERV en pacientes de alto riesgo (UCIs).

Esta norma propone además las medidas epidemiológicas necesarias para prevenir la aparición de resistencia y evitar la diseminación de estas cepas. En los años 1997-1998 se realizó un estudio multicéntrico en hospitales de Santiago, conducido por el Comité de Resistencia de la Sociedad Chilena de Infectología, que investigó la portación intestinal de ERV en 206 pacientes de UCI, encontrándose sólo $2(0,9 \%)$ colonizados con cepas de susceptibilidad intermedia.

En 1999 se comunicó el aislamiento de la primera cepa -estudiada fuera del país- de E faecium resistente a vancomicina, procedente de colonización intestinal, en un paciente del Hospital de Urgencia Asistencia Pública Alejandro del Río, de Santiago. Posteriormente, en el último trimestre del año 2000 se notificaron algunos casos de infecciones por ERV, y tam-

Tabla 3. Enterococcus spp. Características de la resistencia a vancomicina

\begin{tabular}{cccll}
\hline Genotipo & $\begin{array}{l}\text { Vancomicina } \\
\text { CIM }(\boldsymbol{\mu g} / \mathbf{m l})\end{array}$ & $\begin{array}{l}\text { Teicoplanina } \\
\text { CIM }(\boldsymbol{\mu g} / \mathbf{m l})\end{array}$ & $\begin{array}{l}\text { Base } \\
\text { genética }\end{array}$ & Especie \\
\hline Van A & $64>1.000$ & $>16$ & $\begin{array}{l}\text { Plasmidio-cromosomal } \\
\text { Transferible }\end{array}$ & $\begin{array}{l}\text { E. faecalis } \\
\text { E. faecium } \\
\text { E.faecalis }\end{array}$ \\
Van B & $4-1.024$ & $<1$ & $\begin{array}{l}\text { Plasmidio-cromosomal } \\
\text { Transferible }\end{array}$ & $\begin{array}{l}\text { E. faecium } \\
\text { E. gallinarum } \\
\text { E. casseliflavus }\end{array}$ \\
Van C & $2-32$ & $<1$ & $\begin{array}{l}\text { Cromosomal } \\
\text { No transferible }\end{array}$ & $\begin{array}{l}\text { Cromosomal } \text { faecium } \\
\text { No transferible } \\
\text { Van D }\end{array}$ \\
Van E & $64-128$ & $4-8$ & $\begin{array}{l}\text { Cromosomal } \\
\text { No transferible }\end{array}$ & E.faecalis \\
\hline
\end{tabular}




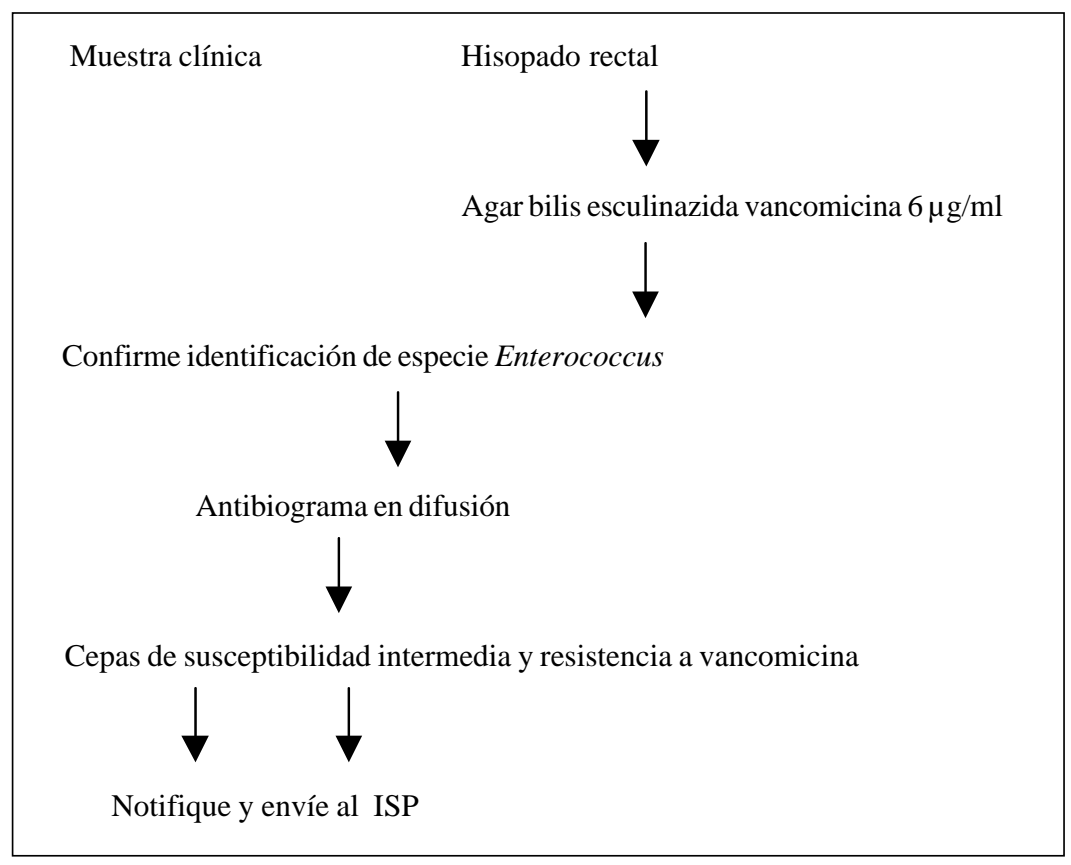

Figura 1. Norma de vigilancia de Enterococcus resistentes a vancomicina (MINSAL, 9.05.2000).

bién de colonizaciones, tanto por E. faecalis como por E. faecium, los que no superan los 10 casos.

Durante el año 2000 el ISP registró una resistencia a vancomicina de $5 \%$ en 97 cepas de E. faecalis y de $29 \%$ en 42 cepas de $E$. faecium, de un total de 145 cepas de Enterococcus referidas de todo Chile a ese centro.

\section{Recomendaciones para estudio de susceptibilidad in vitro de Enterococcus}

Dado que 95\% de los ERV corresponden a E. faecium, es fundamental identificar la especie de Enterococcus, para lo cual se sugiere usar las pruebas fisiológicas indicadas en la Tabla 4.

Tabla 4. Identificación de Enterococcus

$\begin{array}{ll}\text { - Gram } & : \text { cocos Gram positivos, en diplo y cadenas } \\ \text { - Catalasa } & : \text { negativa } \\ \text { - PYR } & : \text { positiva } \\ \text { - Bilis esculina } & : \text { desarrollo de colonias negras } \\ \text { - Na Cl 6,5\% } & : \text { crecimiento } \\ \text { - Telurito } & +\longrightarrow \text { E.faecalis (crecimiento de colonias negras) } \\ & \\ & \end{array}$

Otras pruebas alternativas: movilidad, pigmento, arabinosa, sorbitol. 
Tabla 5. Antimicrobianos a utilizar de rutina en antibiograma de Enterococcus

GRUPO A: Pruebe - Informe

Penicilina o ampicilina

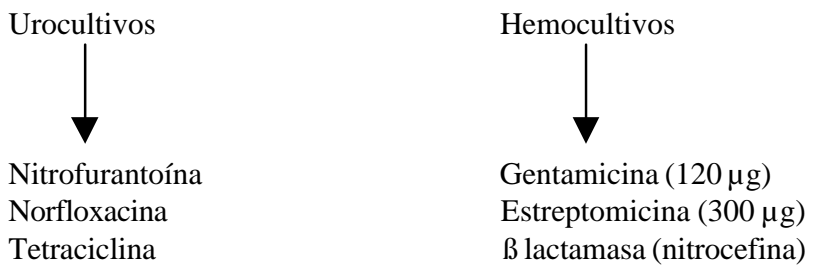

GRUPO B: Pruebe - Informe selectivamente Vancomicina - teicoplanina

Las recomendaciones del NCCLS en el año 2001 indican la realización del antibiograma en difusión con las siguientes especificaciones:

Medio: agar Mueller-Hinton

Inóculo: suspensión directa de colonias o caldo con crecimiento ajustado a 0,5 Mac Farland.

Incubación: $35^{\circ} \mathrm{C}$ durante 16 a 18 hrs excepto para vancomicina que requiere de $24 \mathrm{hrs}$.

Control de calidad: con cepa de Staphylococcus aureus ATCC 2593.

Antimicrobianos a utilizar: se detallan según la muestra clínica (Tabla 5) y la presencia de resistencia a vancomicina (Tabla 6).

Las categorías de susceptibilidad se presentan en la Tabla 7.
Tabla 6. Antibiograma en difusión. Antimicrobianos suplementarios para Enterococcus resistentes a vancomicina

Cloranfenicol

Eritromicina

Tetraciclina

Ciprofloxacina

Quinupristina-dalfopristina

Linezolid

Rifampicina

Tabla 7. Enterococcus spp

Categorías de susceptibilidad NCCLS-2001

\begin{tabular}{lcccccc}
\hline Antimicrobiano & Disco $(\mu \mathrm{g})$ & \multicolumn{2}{c}{ Diámetro $(\mathrm{mm})$} & \multicolumn{2}{c}{ CIM $(\mu \mathrm{g} / \mathrm{ml})$} \\
\hline & & $\mathrm{R}$ & $\mathrm{I}$ & $\mathrm{S}$ & $\mathrm{R}$ & $\mathrm{S}$ \\
Penicilina & $10 \mathrm{UI}$ & $<14$ & - & $>15$ & $>16$ & $<$ \\
Ampicilina & 10 & $<16$ & - & $>17$ & $>16$ & $<$ \\
Ciprofloxacina & 5 & $<15$ & $16-20$ & $>21$ & $>4$ & $<$ \\
Nitrofurantoína & 300 & $<14$ & $15-16$ & $>17$ & $>128$ & $<32$ \\
Tetraciclina & 30 & $<14$ & $15-18$ & $>19$ & $>16$ & $<$ \\
Vancomicina & 30 & $<14$ & $15-16$ & $>17$ & $>32$ & $<$ \\
Teicoplanina & 30 & $<10$ & $11-13$ & $>14$ & $>32$ & $<$ \\
Gentamicina & 120 & 6 & $7-8$ & $>10$ & $>500$ & $<$ \\
Estreptomicina & 300 & 6 & $7-8$ & $>10$ & - & - \\
\hline
\end{tabular}

\section{S 114}


Tabla 8. Sensibilidad de los métodos para la detección de resistencia a vancomicina según fenotipo en Enterococcus spp.*

\begin{tabular}{lccrr}
\hline & $\begin{array}{c}\text { Van A } \\
(\boldsymbol{\%})\end{array}$ & $\begin{array}{c}\text { Van B } \\
(\mathbf{\%})\end{array}$ & $\begin{array}{c}\text { Van C1 } \\
(\boldsymbol{\%})\end{array}$ & $\begin{array}{c}\text { Van C2 } \\
(\mathbf{\%})\end{array}$ \\
\hline Difusión & 100 & 93 & 52 & 63 \\
Epsilometría & 100 & 100 & 100 & 100 \\
Agar Van 6 $\mu \mathrm{g}$ & 100 & 100 & 100 & 100 \\
Mic Scan Co & 100 & 100 & 76 & 7 \\
Mic Scan R & 100 & 53 & 86 & 90 \\
Vitek TA & 100 & 47 & 72 & 67 \\
Vitek 101 & 100 & 100 & 88 & 73 \\
& $*$ & & & \\
\hline
\end{tabular}

Si la cepa demuestra un halo de inhibición intermedio a vancomicina en el antibiograma en difusión o crece en agar screening, vancomicina $6 \mu \mathrm{g} / \mathrm{ml}$, es necesario determinar la CIM a este antibacteriano con método de dilución, $\mathrm{E}$ test ${ }^{\circledR}$ u otro para obtener la confirmación de la resistencia y notificar al Comité de IIH y al ISP. Si no se cuenta con método de dilución, refiera la cepa al ISP. La sensibilidad de los diferentes métodos para detectar la resistencia a vancomicina en Enterococcus se especifica en la Tabla 8 .

\section{BIBLIOGRAFÍA}

1.- MINSAL. Vigilancia, Prevención y Control de Infecciones Intrahospitalarias por Enterococcus resistentes a vancomicina. Circular Minsal 4 C/28 del 09-052001.

2.- Recommendations for Preventing the Spread of Vancomycin Resistance. HICPAC Committee. Morb Mortal Wkly Rep MMWR 1995; 44 (RR12): 1-13.

3.- ELIOPOULOS G M. Vancomycin resistant enterococci. Infect Dis Clin North Am 1997; 11: 851-65.

4.- MURRAY B. Vancomycin resistant enterococci. Am J Med 1997; 101: 284-93.

5.- NCCLS. Performance standards for antimicrobial susceptibility testing: Eleventh International
Supplement. 2001; M100 S11, Vol $21 \mathrm{~N}^{\circ} 1$.

6.- Ministerio de Salud 1999. Informe de Vigilancia de las IIH en Chile.

7.- AUSTIN D J, BONTEN M J, WEINSTEIN R A et al. Vancomycin resistant enterococci in intensive care hospital settings. Proc Natl Acad Sci USA 1999; 96 (12): 6908-13.

8.- CDC. Nosocomial enterococci resistant to vancomycin-United States, 1989-1993. Morb Mortal Wkly Rep MMWR 1993; 42: 597-9.

9.- PINTO M E, GIGLIO M S, GONZÁLEZ P et al. Vigilancia de resistencia a glicopéptidos en $E$. faecum y E. faecalis y otras especies aisladas en muestras fecales de pacientes hospitalizados. Libro de Resúmenes. XIV Congreso Chileno de Infectología, Valdivia, 25 diciembre 1997, CO-37.

10.- JULIET C, FERNÁNDEZ A, TAPIA J C. Vigilancia de resistencia de Enterococcus hospitalarios. Libro de Resúmenes XVII Congreso Chileno de Infectología, Viña del Mar 9 al 12 agosto 2000. CO: 109.

11.- BRENNER P, OTAÍZA F. Resultados de la vigilancia de Enterococco resistente a Vancomicina en Chile. Libro de Resúmenes IX Congreso Chileno de IIH. 2000. CO 10.

12.- STOSOR V, NOSKIN G A, PETERSON L R. The management and prevention of vancomycin resistant enterococci. Infect Med 1996; 13 (6): 487-8, 493-8.

13.- ENDTZ HP, VAN DEN BRAACK A, VAN BELKUM A et al. Comparison of eigth methods to detect vancomycin resistance in Enterococci. J Clin Microbiol 1998; 36: 592-

Correspondencia a:

Chrystal Juliet Larenas

E-mail: chjuliet@yahoo.com 